\section{RMD Open}

Rheumatic \&

Musculoskeletal Diseases

\title{
Environmental and lifestyle factors influencing risk of congenital heart block during pregnancy in anti-Ro/SSA- positive women
}

\author{
Sabrina Meisgen, ${ }^{1}$ Joanna Tingström, ${ }^{1}$ Amanda Skog Andreasson, ${ }^{1}$ \\ Sven-Erik Sonesson, ${ }^{2}$ Ingrid Kockum, ${ }^{3}$ Marie Wahren-Herlenius ${ }^{1}$
}

\begin{abstract}
To cite: Meisgen S, Tingström J Skog Andreasson A, et al. Environmental and lifestyle factors influencing risk of congenital heart block during pregnancy in anti-Ro/SSApositive women. RMD Open 2017;3:e000520. doi:10.1136/ rmdopen-2017-000520
\end{abstract}

- Prepublication history for this paper is available online. To view these files please visit the journal online (http://dx.doi. org/10.1136/rmdopen-2017000520).

SM and JT contributed equally.

Received 13 June 2017 Revised 21 June 2017 Accepted 22 June 2017

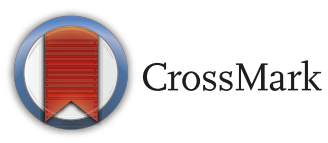

${ }^{1}$ Department of Medicine, Karolinska Institutet, Karolinska University Hospital, Stockholm, Sweden

${ }^{2}$ Department of Women and Child Health, Karolinska Institutet, Karolinska University Hospital, Stockholm, Sweden ${ }^{3}$ Department of Clinical Neuroscience, Karolinska Institutet, Karolinska University Hospital, Stockholm, Sweden

Correspondence to Professor Marie WahrenHerlenius; marie.wahren@ki.se

\section{ABSTRACT}

Objectives Congenital heart block (CHB) occurs in $1 \%-2 \%$ of anti-Ro/SSA antibody-positive pregnancies. A population-based recurrence rate of $12 \%$ indicates that factors other than maternal autoantibodies influence $\mathrm{CHB}$ development. Here we report the first investigation to identify environmental and lifestyle factors influencing the risk of $\mathrm{CHB}$.

Methods A questionnaire focused on environmental and lifestyle factors was distributed to anti-Ro/SSA antibodypositive women who had given birth to at least one child with $\mathrm{CHB}$, and additional data were retrieved from national health registers. Statistical analysis was performed comparing pregnancies resulting in a child with $\mathrm{CHB}$ $(n=81)$ and pregnancies resulting in unaffected siblings $(n=108)$.

Results Analysis of maternal body mass index and weight gain during pregnancy as well as medication intake and sun exposure did not reveal significant differences between CHB-affected and non-CHB pregnancies. By contrast, we found that reports of infections and stressful events were significantly more frequent in CHB-affected pregnancies than in non-CHB affected pregnancies (OR $17.9,95 \% \mathrm{Cl} 4.1$ to $162.8, \mathrm{p}<0.001$ and $\mathrm{OR} 5.5,95 \% \mathrm{Cl} 1.1$ to $55.1, p<0.05$, respectively). Notably, outdoor activity a few hours per day emerged as a protective factor (OR 0.52 , $95 \% \mathrm{Cl} 0.27$ to $0.99, \mathrm{p}<0.05)$. The previously reported factor seasonal timing of pregnancy was confirmed (OR $2.2,95 \% \mathrm{Cl} 1.1$ to $4.2, \mathrm{p}<0.05)$, and multivariate analysis revealed that this association was partly explained by infection and outdoor activity.

Conclusions In this retrospective study, infections, stressful events and time spent with outdoor activities emerged as potential environmental and lifestyle factors influencing the risk of $\mathrm{CHB}$, warranting confirmation in prospective studies.

\section{INTRODUCTION}

Autoimmune congenital heart block (CHB) is a rare but life-threatening condition associated with the presence of anti-Ro/SSA and anti-La/SSB autoantibodies in the mother of the affected child. During pregnancy, maternal antibodies are transferred across

\section{Key messages}

What is already known about this subject?

- This is the first report on environmental and lifestyle factors influencing the risk of congenital heart block (CHB).

What does this study add?

- Infections, stressful events and time spent with outdoor activities emerged as environmental and lifestyle factors influencing the risk of CHB.

How might this impact on clinical practice?

- The data contribute towards elucidating the pathogenic disease mechanism and may improve counselling for women at risk of giving birth to a child affected by CHB.

the placenta and may contribute to an inflammatory reaction in the fetal heart that will result in a permanent block of electric signal conduction at the atrioventricular node. CHB usually develops between weeks 18 and 24 of pregnancy and is often first detected as the fetus presents with signs of bradycardia. ${ }^{1-4}$

The association between CHB and the presence of maternal anti-Ro/SSA autoantibodies is strong, with anti-Ro/SSA antibodies detected in close to $90 \%$ of affected pregnancies. ${ }^{5}$ However, recurrence rates of $12 \%$ despite the continued presence of maternal antibodies $^{6-8}$ indicate that additional factors contribute to disease pathogenesis and are required for $\mathrm{CHB}$ development. Fetal genetics, in particular the HLA locus, have been implicated in susceptibility to $\mathrm{CHB}$ in recent studies. ${ }^{910}$ In addition, hypothyroidism and maternal age have been suggested as risk factors for CHB. Parity and fetal sex, however, do not appear to affect CHB risk. ${ }^{611-14}$

Notably, no study so far has investigated the potential influence of lifestyle factors on the 
risk for $\mathrm{CHB}$, although we recently observed that season of birth was associated with CHB occurrence. ${ }^{6}$ To identify environmental and lifestyle factors that may influence CHB development, we used data from the Swedish Medical Birth Register and performed the present questionnaire-based study, inviting anti-Ro/SSA antibody-positive mothers with at least one child with CHB and included in a population-based cohort to participate.

\section{PATIENTS AND METHODS}

\section{Participants}

Identification of a population-based cohort of anti-Ro/ SSA antibody-positive women giving birth to at least one child with CHB was described previously. ${ }^{15}$ All women in the cohort having given birth to a child between the years 1959 and 2009 were invited to participate $(n=88)$. The study was approved by the Regional Ethical Committee Stockholm, and the women gave informed written consent.

\section{Questionnaire}

A questionnaire was generated based on data from interviews conducted in a subgroup of women from this cohort $^{16}$ and included demographic questions, questions regarding maternal health and medication as well as questions focusing on lifestyle factors before and during pregnancy. Space was provided for reporting optional additional information. Data collection was conducted between March and June 2011. The response rate was $89 \%(\mathrm{n}=78 / 88)$. However, all women did not respond to all questions in the questionnaire, and the number of respondents for each question is therefore indicated in the tables presenting the data. Each woman participating in the study was requested to answer a set of questions for each of her pregnancies-the one leading to the birth of a child with CHB as well as the one(s) resulting in the birth of a healthy sibling, and responses were compared between CHB and non-CHB pregnancies. For each question, the time interval before or during pregnancy to which the answer should refer was clearly stated. Time intervals of the investigated factors are detailed in the table footnotes.

\section{Data from the Swedish Medical Birth Register}

Data on maternal height, weight and weight gain during pregnancy were extracted from the national Swedish Medical Birth Register (www.socialstyrelsen.se). Data for body mass index (BMI) and gestational weight increase were available for a limited number of pregnancies as indicated in the tables.

\section{Statistical analysis}

The Mann-Whitney U test (GraphPad Prism 5) was applied to assess differences in maternal BMI and weight increase during pregnancy. $\chi^{2}$ statistics were used to calculate differences between the two pregnancy outcome groups using the Fisher's exact test (R, V.2.15.2). Haldane's correction was used for OR calculations with zero counts in one of the groups. ${ }^{17}$ Logistic regression analysis using the generalised linear model in R V.2.15.2) with a binominal distribution was used for multivariate analyses. For the multivariate analysis, combined counts from the categories a few hours/day and a few hours/ week were used for the variable outdoor activity. Testing for trends regarding lifestyle factors was performed with the Cochrane-Armitage test in $\mathrm{R}$ (V.2.15.2). A p value $<0.05$ was considered significant. We have previously reported data on season of birth from 69 of the cases included in the present study. ${ }^{6}$

\section{RESULTS}

\section{Response rate}

To identify environmental and lifestyle factors that may influence CHB development, we designed the present questionnaire-based study. Anti-Ro/SSA antibody-positive women included in a population-based cohort of mothers who had given birth to at least one child with $\mathrm{CHB}^{15}(\mathrm{n}=88)$ were invited to participate. The response rate to the questionnaire was $89 \%(n=78 / 88)$, which generated information on 81 pregnancies where the child developed CHB and 108 sibling pregnancies where the child did not develop CHB.

\section{Maternal rheumatic diagnosis and autoantibody status}

All women participating in the present study were anti-Ro/SSA antibody positive. Of these, $100 \%$ and $76 \%$ had antibodies to the Ro52 and Ro60 component of the Ro/SSA antigen, respectively, and $67 \%$ were anti-La/SSB antibody-positive at the time the study was conducted (table 1). At the time of CHB, $58 \%$ of the women had not been diagnosed with a rheumatic disease or were asymptomatic. Women reporting a rheumatic diagnosis most commonly had systemic lupus erythematosus (SLE) or Sjögren's syndrome, while 4\% of the women reported a diagnosis of undifferentiated connective tissue disease (UCTD) (table 1).

\section{Table 1 Characteristics of the participating women}

Rheumatic diagnosis* $(n=78)^{\dagger}$

\begin{tabular}{lr} 
SLE & $21 \%$ \\
Sjögren's syndrome & $18 \%$ \\
UCTD & $4 \%$ \\
No diagnosis/asymptomatic & $57 \%$ \\
Anti-Ro/SSA and anti-La/SSB antibodies $(\mathrm{n}=78)^{\dagger}$ & \\
Anti-Ro52 $^{\text {Anti-Ro60 }}$ & $100 \%$ \\
Anti-La $^{*}$ & $76 \%$ \\
Smokers $^{\star}(\mathrm{n}=76)^{\ddagger}$ & $67 \%$ \\
Ever $^{\text {Never }}$ & \\
\hline
\end{tabular}

*Self-reported.

†Number of participating women.

$\ddagger$ Number of women responding to the question.

SLE, systemic lupus erythematosus; UCTD, undifferentiated connective tissue disease. 
Table 2 Maternal BMI and maternal weight increase during pregnancy

\begin{tabular}{llll}
\hline & $\begin{array}{l}\text { CHB } \\
\text { pregnancies }\end{array}$ & $\begin{array}{l}\text { Non-CHB } \\
\text { pregnancies }\end{array}$ & $\begin{array}{l}\mathbf{p} \\
\text { Value }\end{array}$ \\
\hline $\mathrm{BMI}^{*}$ & & & \\
$\mathrm{n}^{\dagger}$ & 22 & 34 & \\
$\quad\left(\right.$ mean $\left.\pm 1 \mathrm{SD}, \mathrm{kg} / \mathrm{m}^{2}\right)$ & $23.2 \pm 3.1$ & $23.1 \pm 3.9$ & n.s. \\
$\begin{array}{l}\text { Gestational weight } \\
\text { increase }\end{array}$ & & & \\
$\mathrm{n}^{\dagger}$ & 23 & 42 & \\
$\quad($ mean $\pm 1 \mathrm{SD}, \mathrm{kg})$ & $11.2 \pm 5.9$ & $10.7 \pm 6.0$ & n.s. \\
\hline
\end{tabular}

${ }^{*}$ Before pregnancy.

†Number of pregnancies where information was available from the Swedish Medical Birth Register.

$\mathrm{BMI}$, body mass index; CHB, congenital heart block.

The proportion of ever and never smokers among the women were almost equal, with $49 \%$ of ever versus $51 \%$ of never smokers (table 1). Among women who reported being ever-smokers, $19 \%(n=15)$ stated that they smoked during pregnancy, and they reported similar smoking habits for both their CHB-affected and non-CHB-affected pregnancies (data not shown).

\section{Maternal BMI and weight increase during pregnancy}

Neither maternal BMI nor weight increase during pregnancy significantly differed between the CHB and non-CHB pregnancy groups (table 2). Mean maternal BMI before pregnancy was approximately 23 for both groups, and the CHB-affected and non-affected pregnancies' mean maternal weight increase during pregnancy was $11.2 \pm 5.9 \mathrm{~kg}$ and $10.7 \pm 6.0 \mathrm{~kg}$, respectively (table 2 ).

\section{Maternal medication}

Investigating the maternal intake of medication related to treatment of rheumatic diseases 3 months before pregnancy and up until gestational week 25, we found that few women indicated taking any immunomodulatory medication, consistent with the large proportion of women not diagnosed with any rheumatic disease at the time of pregnancy. Exposure to other drugs was not systematically recorded. We did not observe any significant difference in medication intake reports between the CHB and non-CHB-affected pregnancies (table 3). In the CHB pregnancy group, $19 \%$ and $15 \%$ of women reported taking immunomodulatory medication before and during pregnancy, respectively, while $13 \%$ and $11 \%$ reported so in the non-CHB pregnancy group, respectively (table 3 ).

\section{Lifestyle factors and infections}

In a previous study, we found that season of birth was significantly associated with $\mathrm{CHB}$, with an increased proportion of children with CHB being born during the Swedish summer. This in turn corresponds to the CHB risk period (gestational weeks 18-24) occurring during the months of January to March. ${ }^{6}$ Seasonal timing
Table 3 Medication before and during pregnancy in antiRo/SSA-positive women.

\begin{tabular}{|c|c|c|}
\hline & $\begin{array}{l}\text { CHB } \\
\text { pregnancy, \% } \\
\text { (frequency) }\end{array}$ & $\begin{array}{l}\text { Non-CHB } \\
\text { pregnancy, \% } \\
\text { (frequency) }\end{array}$ \\
\hline Before pregnancy ${ }^{*}$ & $19(15 / 80)$ & $13(13 / 103)$ \\
\hline Acetylsalicylic acid & $4(3 / 80)$ & $5(5 / 103)$ \\
\hline Hydroxychloroquine & $6(5 / 80)$ & $3(3 / 103)$ \\
\hline Steroids (prednisolone) & $5(4 / 80)$ & $3(3 / 103)$ \\
\hline DMARDs $^{\dagger}$ & $1(1 / 80)$ & $1(1 / 103)$ \\
\hline Levotyroxin & $3(2 / 80)$ & $1(1 / 103)$ \\
\hline During pregnancy ${ }^{\ddagger}$ & $15(12 / 80)$ & $11(11 / 103)$ \\
\hline Acetylsalicylic acid & $3(2 / 80)$ & $4(4 / 103)$ \\
\hline Hydroxychloroquine & $1(1 / 80)$ & $2(2 / 103)$ \\
\hline Steroids (prednisolone) & $8(6 / 80)$ & $4(4 / 103)$ \\
\hline DMARDs $^{\dagger}$ & $1(1 / 80)$ & $0(0 / 103)$ \\
\hline Levotyroxin & $3(2 / 80)$ & $0(0 / 103)$ \\
\hline $\begin{array}{l}\text { Intravenous } \\
\text { immunoglobulin }\end{array}$ & $0(0 / 80)$ & $1(1 / 103)$ \\
\hline
\end{tabular}

*Within 3 months before pregnancy and up until week 25 of gestation.

†Disease-modifying antirheumatic drugs (DMARDs): azathioprine and cyclosporine.

fUntil gestational week 25. No comparisons between $\mathrm{CHB}$ and non-CHB groups were significant ( $p>0.05)$.

of pregnancy may influence the amount of time spent outdoors in climates such as in Sweden, thereby potentially impacting sun exposure and physical activity. We therefore asked the women in our cohort to indicate how much outdoor activity they were engaged in while pregnant as well as their sun exposure habits (table 4). We observed that high outdoor activity (a few hours per day) was protective (OR $0.52,95 \% \mathrm{CI} 0.27$ to $0.99, \mathrm{p}=0.04$, table 4). The women reported spending a few hours per day in outdoor activities during $48 \%$ of pregnancies leading to a CHB-affected child versus $64 \%$ of the pregnancies resulting in a child without CHB. Interestingly, we found a significant trend between decreasing outdoor activity and CHB pregnancy outcomes $\left(\mathrm{p}^{-}{ }_{\text {Trend }}=0.01\right.$, table 4).

Most women did not report any change in their sun exposure habits during pregnancy; a decrease or increase in sun exposure was reported during $21 \%-24 \%$ and $2 \%-5 \%$ of pregnancies, respectively. We did not observe any significant difference in sun exposure habits between pregnancies in which the child developed or did not develop CHB (table 4).

Infections are more common during the cold season, and an association between seasonal timing of pregnancy and $\mathrm{CHB}$ might therefore reflect a link to infections. The women in this cohort reported having an infection (respiratory tract infection or influenza) before gestational week 25 in $28 \%$ of the pregnancies resulting in a child with $\mathrm{CHB}$ versus only $2 \%$ of the pregnancies 
Table 4 Lifestyle factors before and during pregnancy in anti-Ro/SSA positive women

\begin{tabular}{|c|c|c|c|c|c|}
\hline & $\begin{array}{l}\text { CHB } \\
\text { pregnancies }\end{array}$ & $\begin{array}{l}\text { Non-CHB } \\
\text { pregnancies }\end{array}$ & OR $(95 \% \mathrm{Cl})$ & p Value & p Trend \\
\hline \multicolumn{5}{|c|}{ I. Outdoor activity during daytime ${ }^{\star}, \dagger, \%$ (frequency) } & 0.01 \\
\hline A few hours/day & $48(37 / 77)$ & $64(64 / 100)$ & $0.52(0.27$ to 0.99$)$ & 0.04 & \\
\hline A few hours/week & $43(33 / 77)$ & $31(31 / 100)$ & $1.66(0.85$ to 3.25$)$ & 0.12 & \\
\hline A few hours/month or never $\ddagger$ & $9(7 / 77)$ & $5(5 / 100)$ & 1.89 (0.49 to 7.90$)$ & 0.37 & \\
\hline \multicolumn{6}{|l|}{ II. Sun exposure*, \% (frequency) } \\
\hline Increased sun exposure & $5(4 / 79)$ & $2(2 / 108)$ & 2.81 (0.39 to 31.82$)$ & n.s & \\
\hline Decreased sun exposure & $21(16 / 76)$ & $24(25 / 106)$ & 0.86 (0.39 to 1.86$)$ & n.s & \\
\hline Sunbathing outside & & & & & n.s \\
\hline daily & $13(10 / 79)$ & $15(16 / 106)$ & $0.82(0.31$ to 2.05$)$ & n.s & \\
\hline regularly & $27(21 / 79)$ & $25(26 / 106)$ & 1.11 (0.53 to 2.28$)$ & n.s & \\
\hline sparsely & $37(29 / 79)$ & $37(39 / 106)$ & $1.00(0.51$ to 1.90$)$ & n.s & \\
\hline never & $24(19 / 79)$ & $24(25 / 106)$ & $1.03(0.48$ to 2.14$)$ & n.s & \\
\hline Solarium & & & & & n.s \\
\hline A few times /week & $0(0 / 80)$ & $1(1 / 108)$ & $0.67(0.01 \text { to } 13.04)^{\S}$ & n.s & \\
\hline A few times/month & $5(4 / 80)$ & $5(5 / 108)$ & $1.08(0.20$ to 5.22$)$ & n.s & \\
\hline never & $95(76 / 80)$ & $95(102 / 108)$ & $1.12(0.25$ to 5.58$)$ & n.s & \\
\hline $\begin{array}{l}\text { Trip abroad with increased sun } \\
\text { exposure }\end{array}$ & $10(8 / 77)$ & $8(9 / 108)$ & $1.27(0.40$ to 3.93$)$ & n.s & \\
\hline \multicolumn{6}{|c|}{ III. Infections* and stressful events*, \% (frequency) } \\
\hline Infectious diseaseף & $28(21 / 76)$ & $2(2 / 97)$ & $17.87(4.11$ to 162.84$)$ & 0.00013 & \\
\hline Stressful event ${ }^{\star \star}$ & $11(8 / 76)$ & $2(2 / 97)$ & 5.54 (1.05 to 55.08$)$ & 0.02 & \\
\hline
\end{tabular}

${ }^{*}$ Before week 25 of pregnancy,

†Examples given were games, sports, walks, gardening, work.

‡Categories few hours/month and never were combined in the table; none answered never.

$\S$ Haldane's correction was used for calculation.

ףIncludes respiratory tract infections, gastrointestinal tract infections and skin infections.

${ }^{\star}$ Death or severe disease of a close relative or own severe disease.

$\mathrm{CHB}$, congenital heart block.

resulting in a child without $\mathrm{CHB}$, leading to a significant association between self-reported infection and CHB development (OR 17.87, 95\% CI 4.11 to 162.84 , p $<0.001$, table 4).

\section{Psychological stress week 25}

In a semiopen question, the women were asked if they had experienced any particular distressing event during their pregnancy before week 25. A positive answer was given for $11 \%(8 / 76)$ of the pregnancies in which the child developed CHB versus only 2\% (2/97) of the pregnancies resulting in a sibling without CHB (OR 5.54, $95 \%$ CI 1.05 to $55.08, p=0.02$, table 4 ). For six of the CHB pregnancies, this 'distressing event' was reported to be the death or severe disease of a close relative. The additional two positive answers related to a cancer diagnosis and severe depression of the women themselves. For the non-CHB pregnancies, one woman reported that a close relative had died, and the other indicated that she herself was hospitalised due to a severe unspecified diagnosis.
Multivariate analysis of risk factors in CHB development

Infection and outdoor activity emerged as factors potentially associated with CHB from our analysis. Since it is likely that both of these parameters are tied to seasonal variation, a factor that we have previously shown to be associated with CHB occurrence, we performed a multivariate logistic regression analysis to investigate if these factors were independently associated with $\mathrm{CHB}$. We found that infection was significantly associated with $\mathrm{CHB}$ in a univariate analysis (OR 18.2, 95\% CI 5.0 to 116.1, $\mathrm{p}<0.001$ ) (table 5) and that this association remained significant in the multivariate analysis when seasonal timing of pregnancy and outdoor activity were taken into account (OR 18.0, 95\% CI 4.8 to $117.2, \mathrm{p}<0.001$ ) (table 5). Although outdoor activity did not significantly associate with CHB in the univariate analysis, we found it positively modulated pregnancy outcome when infections were taken into account, as indicated by an OR of 0.5 for CHB development ( $95 \%$ CI 0.2 to $0.9, \mathrm{p}<0.05)$ in multivariate analysis (table 5). Notably, the previously reported association of season of birth with $\mathrm{CHB}$ was also 
Table 5 Multivariate association analysis of risk factors for $\mathrm{CHB}$ outcome

\begin{tabular}{|c|c|c|c|c|}
\hline Variable & Covariate 1 & Covariate 2 & OR $(95 \% \mathrm{Cl})$ & p Value \\
\hline Infection* & & & 18.2 (5.0 to 116.1$)$ & 0.00013 \\
\hline Infection & Birth season $^{\dagger}$ & & 17.3 (4.7 to 111.3$)$ & 0.00018 \\
\hline Infection & & Outdoor activity $^{\ddagger}$ & 18.2 (4.9 to 118.2$)$ & 0.00016 \\
\hline Infection & Birth season & Outdoor activity & 18.0 (4.8 to 117.2$)$ & 0.00018 \\
\hline Outdoor activity & & & $0.6(0.3$ to 1.1$)$ & 0.07 \\
\hline Outdoor activity & Infection & & $0.5(0.2$ to 0.9$)$ & 0.04 \\
\hline Outdoor activity & & Birth season & 0.6 (0.3 to 1.2$)$ & 0.14 \\
\hline Outdoor activity & Infection & Birth season & 0.5 (0.2 to 1.0$)$ & 0.07 \\
\hline Birth season & & & 2.2 (1.1 to 4.2$)$ & 0.02 \\
\hline Birth season & & Infection & 1.9 (0.9 to 3.9) & 0.08 \\
\hline Birth season & Outdoor activity & & $1.9(0.9$ to 3.7$)$ & 0.07 \\
\hline Birth season & Outdoor activity & Infection & $1.6(0.7$ to 3.5$)$ & 0.20 \\
\hline
\end{tabular}

*Includes respiratory tract infections, gastrointestinal tract infections and skin infections.

†CHB risk period falls into January-March. ${ }^{6}$

$\ddagger$ Examples given were games, sports, walks, gardening and work.

$\mathrm{CHB}$, congenital heart block.

detected in the present study (OR 2.2, 95\% CI 1.1 to 4.2, $\mathrm{p}<0.05$, univariate analysis) (table 5 ), but when adjusting for infection or outdoor activity, the significance was lost (OR $1.9,95 \%$ CI 0.9 to $3.9, \mathrm{p}=0.08$, and OR $1.9,95 \% \mathrm{CI}$ 0.9 to $3.7, \mathrm{p}=0.07$, respectively, in multivariate analysis) (table 5). This indicates that the association observed between seasonal timing of pregnancy and CHB development is in part explained by the risk conferred by infection and lack of outdoor activity.

\section{DISCUSSION}

Given the relatively low recurrence rate of anti-Ro/SSA autoantibody-associated CHB despite persisting maternal autoantibodies, genetic and environmental factors have been suggested to play a role in disease development. However, which environmental factors might potentially influence pregnancy outcome remains largely unknown. We therefore performed the present questionnaire-based study to identify possible environmental and lifestyle factors associated with CHB in a population-based cohort of women who had given birth to at least one child with CHB.

Analysing potential risk factors for $\mathrm{CHB}$ related to maternal features, we found that neither BMI nor weight increase during pregnancy significantly differed between pregnancies resulting in a child with $\mathrm{CHB}$ and pregnancies resulting in a sibling without $\mathrm{CHB}$, although the number of analysed pregnancies was limited due to data availability for these factors. However, as the maternal BMIs were predominantly within the normal range in the studied cohort, we cannot exclude that more extreme BMIs or weight changes during pregnancy might yet affect CHB development.

Smoking is known to promote the development of autoimmune and inflammatory conditions, ${ }^{18}$ and in Sweden women are actively recommended to avoid smoking during pregnancy. Our cohort nevertheless included several women who reported smoking during pregnancy. However, as these women smoked during each of their pregnancies, we did not observe any difference between CHB-affected and non-CHB-affected pregnancies regarding smoking habits. While these data might suggest that smoking does not play a central role in CHB development, it might be worth investigating the potential impact of smoking on CHB development in other cohorts. However, the rarity of CHB (1 in 23000 births $^{19}$ ) combined with the general recommendation for women to refrain from smoking during pregnancy would likely render such a study difficult to conduct.

Investigation of maternal medication intake 3 months before pregnancy and up until gestational week 25 did not reveal any significant differences between pregnancies where the child developed CHB and unaffected pregnancies. Hydroxychloroquine has been proposed to reduce neonatal morbidity and the recurrence risk of $\mathrm{CHB}^{2021}$ in patients with SLE. In the present study, we did not observe any significant protective effect of hydroxychloroquine. However, there were few pregnancies during which women reported taking such medication (nine women before and three during pregnancy), which likely limited the possibility to detect any potential association with CHB. Of note, only medication prescribed for the maternal rheumatic disease was investigated, and we can therefore not exclude that other medication may impact pregnancy outcome in terms of CHB.

Occurrence of a common infection during pregnancy, such as infection of the respiratory tract or influenza, was the variable most significantly associated with $\mathrm{CHB}$ development in our cohort. While this finding needs replication, ideally in a prospective study to avoid influence of 
recall bias, it is an interesting hypothesis that infection may trigger the maternal inflammatory state, which in turn could contribute to CHB development.

We have previously found that CHB is associated with season of birth, ${ }^{6}$ more specifically with the pregnancy risk period (weeks 18-24) falling during the Swedish winter (January-March). Such an association might be due to the increased frequency of infections during winter. We indeed detected an association between $\mathrm{CHB}$ and the mother reporting an infection up until pregnancy week 25 in the present study, and multivariate analysis revealed that the association between CHB and season of birth was not independent of infection, altogether suggesting that infections might represent an important factor underlying the association between $\mathrm{CHB}$ and season of birth. Importantly, however, seasonal variation also influences other factors, such as vitamin D levels ${ }^{6}$ and time spent outdoors. We therefore investigated the lifestyle factors outdoor activity and sun exposure in our cohort. Although the women did not report any difference in sun exposure habits during CHB-affected and non-CHB-affected pregnancies, we observed that they reported more time spent in outdoor activities during pregnancies resulting in a child without CHB than during pregnancies resulting in a child with $\mathrm{CHB}$. Notably, multivariate analysis showed that the association between $\mathrm{CHB}$ and season of birth was not independent of outdoor activity, suggesting that outdoor activity might represent another important factor besides infections underlying the association between CHB and season of birth. While time spent in outdoor activities could simply reflect the women's general well-being during pregnancy, it may also indicate an increased exposure to UV light and therefore be linked to higher vitamin D levels. Importantly, vitamin D plays an important role in regulating innate and adaptive immunity and has been reported to modulate placental inflammation. ${ }^{22} 23$ Increased time spent in outdoor activities may also indicate more physical activity (examples of outdoor activity given in our questionnaire comprised activities involving mild-to-moderate physical effort such as gardening and walking).

A few of the women in our cohort reported having experienced a psychologically stressful event, such as the death or severe disease of a close relative, during their pregnancy up until week 25. While such a report was significantly more frequent for CHB-affected pregnancies than for pregnancies resulting in a child without CHB, these findings need to be confirmed in larger cohorts. Experiencing a life-changing event leading to psychological stress has previously been reported to negatively influence pregnancy outcome. ${ }^{24}{ }^{25}$ Psychological stress may therefore be a factor of potential interest in relation to pregnancy outcome in anti-Ro/SSA antibody-positive women.

This questionnaire-based study was performed retrospectively, and limitations therefore include recall biases linked to the time elapsed since the women were pregnant as well as the fact that knowledge of pregnancy outcome may have affected responses to the questionnaire. However, there is a substantial lack of data as to what environmental factors might influence pregnancy outcome in anti-Ro/SSA antibody-positive women and, given the rarity of the disease, there are very few cohorts available to investigate such factors. We therefore believe that, despite its limitations, our study generates novel insights into what risk factors may warrant further investigation in other, ideally prospective, cohorts.

In summary, we here report the first study of environmental and lifestyle factors that may influence $\mathrm{CHB}$ development in anti-Ro/SSA antibody-exposed fetuses. Novel factors including infections and stressful events were associated with an increased risk for $\mathrm{CHB}$, while outdoor activity appeared protective, warranting further investigation in prospective studies. Identification of external elements associated with CHB development may contribute to elucidating the pathogenic disease mechanisms and improve counselling for women at risk of giving birth to a child affected by CHB.

Acknowledgements We would like to thank Aurélie Ambrosi, Karolinska Institutet, for discussions and excellent support in writing the manuscript.

Contributors JT and MW-H conceived the study; JT collected data that was analysed by SM, AS and JT together with IK, S-ES and MW-H. SM, JT and AS wrote the manuscript; S-ES, IK and MW-H participated in revision until its final form.

Funding This study was supported by grants from the Swedish Research Council; the Heart-Lung Foundation; the Stockholm County Council; the Karolinska Institute; the Swedish Rheumatism Association; King Gustaf the Vth 80-year Foundation; the Freemason Children Foundation Stockholm; and the Torsten and Ragnar Söderberg Foundation.

Competing interests None declared.

Patient consent Obtained.

Ethics approval The Regional Ethical Committee, Stockholm.

Provenance and peer review Not commissioned; externally peer reviewed.

Open Access This is an Open Access article distributed in accordance with the Creative Commons Attribution Non Commercial (CC BY-NC 4.0) license, which permits others to distribute, remix, adapt, build upon this work non-commercially, and license their derivative works on different terms, provided the original work is properly cited and the use is non-commercial. See: http://creativecommons.org/ licenses/by-nc/4.0/

(C) Article author(s) (or their employer(s) unless otherwise stated in the text of the article) 2017. All rights reserved. No commercial use is permitted unless otherwise expressly granted.

\section{REFERENCES}

1. Buyon JP, Ben-Chetrit E, Karp S, et al. Acquired congenital heart block. pattern of maternal antibody response to biochemically defined antigens of the SSA/Ro-SSB/La system in neonatal lupus. $J$ Clin Invest 1989;84:627-34.

2. McCauliffe DP. Neonatal lupus erythematosus: a transplacentally acquired autoimmune disorder. Semin Dermatol 1995;14:47-53.

3. Salomonsson S, Strandberg L. Autoantibodies associated with congenital heart block. Scand J Immunol 2010;72:185-8.

4. Brucato A, Doria A, Frassi M, et al. Pregnancy outcome in 100 women with autoimmune diseases and anti-Ro/SSA antibodies: a prospective controlled study. Lupus 2002;11:716-21.

5. Brito-Zerón P, Izmirly PM, Ramos-Casals M, et al. The clinical spectrum of autoimmune congenital heart block. Nat Rev Rheumatol 2015;11:301-12.

6. Ambrosi A, Salomonsson S, Eliasson $\mathrm{H}$, et al. Development of heart block in children of SSA/SSB-autoantibody-positive women is associated with maternal age and displays a season-of-birth pattern. Ann Rheum Dis 2012;71:334-40. 
7. Julkunen $\mathrm{H}$, Eronen $\mathrm{M}$. The rate of recurrence of isolated congenital heart block: a population-based study. Arthritis Rheum 2001;44:487-8.

8. Llanos C, Izmirly PM, Katholi M, et al. Recurrence rates of cardiac manifestations associated with neonatal lupus and maternal/fetal risk factors. Arthritis Rheum 2009;60:3091-7.

9. Clancy RM, Marion MC, Kaufman KM, et al. Identification of candidate loci at 6 p21 and 21q22 in a genome-wide association study of cardiac manifestations of neonatal lupus. Arthritis Rheum 2010;62:3415-24.

10. Meisgen S, Östberg T, Salomonsson S, et al. The HLA locus contains novel foetal susceptibility alleles for congenital heart block with significant paternal influence. J Intern Med 2014;275:640-51.

11. Solomon DG, Rupel A, Buyon JP. Birth order, gender and recurrence rate in autoantibody-associated congenital heart block: implications for pathogenesis and family counseling. Lupus 2003;12:646-7.

12. Izmirly PM, Kim MY, Llanos C, et al. Evaluation of the risk of anti-SSA/Ro-SSB/La antibody-associated cardiac manifestations of neonatal lupus in fetuses of mothers with systemic lupus erythematosus exposed to hydroxychloroquine. Ann Rheum Dis 2010;69:1827-30.

13. Spence D, Hornberger L, Hamilton R, et al. Increased risk of complete congenital heart block in infants born to women with hypothyroidism and anti-Ro and/or anti-La antibodies. J Rheumatol 2006;331:167-70.

14. Askanase AD, Iloh I, Buyon JP. Hypothyroidism and antithyroglobulin and antithyroperoxidase antibodies in the pathogenesis of autoimmune associated congenital heart block. J Rheumatol 2006;33:10.

15. Salomonsson S, Dzikaite V, Zeffer E, et al. A population-based investigation of the autoantibody profile in mothers of children with atrioventricular block. Scand J Immunol 2011;74:511-7.
16. Tingström J, Henriksson EW, Sonesson SE, et al. Ro52 autoantibody-positive women's experience of being pregnant and giving birth to a child with congenital heart block. Midwifery 2013;29:18-23

17. HALDANE JB. The estimation and significance of the logarithm of a ratio of frequencies. Ann Hum Genet 1956;20:309-11.

18. Klareskog L, Padyukov L, Alfredsson L. Smoking as a trigger for inflammatory rheumatic diseases. Curr Opin Rheumatol 2007;19:49-54.

19. Skog A, Lagnefeldt L, Conner P, et al. Outcome in 212 anti-Ro/ SSA-positive pregnancies and population-based incidence of congenital heart block. Acta Obstet Gynecol Scand 2016;95:98-105.

20. Leroux M, Desveaux C, Parcevaux M, et al. Impact of hydroxychloroquine on preterm delivery and intrauterine growth restriction in pregnant women with systemic lupus erythematosus: a descriptive cohort study. Lupus 2015;24:1384-91.

21. Izmirly PM, Costedoat-Chalumeau N, Pisoni CN, et al. Maternal use of hydroxychloroquine is associated with a reduced risk of recurrent anti-SSA/Ro-antibody-associated cardiac manifestations of neonatal lupus. Circulation 2012;126:76-82.

22. Lagishetty V, Liu NQ, Hewison M. Vitamin D metabolism and innate immunity. Mol Cell Endocrinol 2011;347:97-105.

23. Liu NQ, Kaplan AT, Lagishetty V, et al. Vitamin D and the regulation of placental inflammation. J Immunol 2011;186:5968-74.

24. Adam Y, Meinlschmidt G, Lieb R. Associations between mental disorders and the common cold in adults: a population-based crosssectional study. J Psychosom Res 2013;74:69-73.

25. László KD, Liu XQ, Svensson T, et al. Psychosocial stress related to the loss of a close relative the year before or during pregnancy and risk of preeclampsia. Hypertension 2013;62:183-9. 